\title{
Gödel on Intuition and on Hilbert's finitism
}

\author{
W. W. Tait*
}

There are some puzzles about Gödel's published and unpublished remarks concerning finitism that have led some commentators to believe that his conception of it was unstable, that he oscillated back and forth between different accounts of it. I want to discuss these puzzles and argue that, on the contrary, Gödel's writings represent a smooth evolution, with just one rather small double-reversal, of his view of finitism. He used the term "finit" (in German) or "finitary" or "finitistic" primarily to refer to Hilbert's conception of finitary mathematics. On two occasions (only, as far as I know), the lecture notes for his lecture at Zilsel's [Gödel, 1938a] and the lecture notes for a lecture at Yale [Gödel, *1941], he used it in a way that he knew - in the second case, explicitly — went beyond what Hilbert meant.

Early in his career, he believed that finitism (in Hilbert's sense) is openended, in the sense that no correct formal system can be known to formalize all finitist proofs and, in particular, all possible finitist proofs of consistency of first-order number theory, $P A$; but starting in the Dialectica paper [Gödel, 1958], he expressed in writing the view that $\epsilon_{0}$ is an upper bound on the finitist ordinals, and that, therefore, the consistency of $P A$, cannot be finitistically proved. Although I do not understand the "therefore" (see $\S 8$ below), here was a genuine change in his views. But I am unaware of any writings in which he retracted this new position. Incidentally, the analysis he gives of what should count as a finitist ordinal in [Gödel, 1958; Gödel, 1972] should in fact lead to the bound $\omega^{\omega}$, the ordinal of primitive recursive arithmetic, PRA. (Again, see $\S 8$ below.) The one area of double-reversal in the development of his ideas concerns the view, expressed in letters to Bernays in the early 196o's, about whether or not $\epsilon_{0}$ is the least upper bound on the finitist ordinals. (See $\S 1$ below.)

*I have had valuable comments from Michael Friedman, Wilfried Sieg, Daniel Sutherlandand members of the editorial board on earlier versions of this paper, and I thank all of them. 
There is a second theme I will pursue in this paper as well, namely Gödel's notion of Anschauung, that he takes to be the basis of Hilbert's finitism, and its relation both to Kant's and to that of Hilbert and Bernays. The term is translated as 'intuition' from Gödel's works in German, but he himself translated it explicitly as concrete intuition in [1972] and identified it both with Kant's Anchauung and with the concept of intuition underlying Hilbert's finitism. I believe, however, that Gödel's notion of intuition, whatever relationship it bears to Hilbert's, is very different from Kant's and that the latter's notion of intuition and the conception of arithmetic that most naturally follows from his admittedly somewhat meager discussion of the subject lead quite naturally in fact to the arithmetic formalizable in $P R A$, thus differing both from Gödel's early view that finitism (i.e. the mathematics based on intuitive evidence) is unbounded and from the bound $\epsilon_{0}$ that he later entertained for the finitist ordinals.

1. Gödel expressed the view that finitism is open-ended from almost the beginning of his career: In a well-known passage in his 1931 incompleteness paper, he wrote that his incompleteness theorems

do not contradict Hilbert's formalistic standpoint. For this standpoint presupposes only the existence of a consistency proof in which nothing but finitary means of proof is used, and it is conceivable that there exist finitary proofs that cannot be expressed in the formalism of [simple type theory over the natural numbers].[1931, p. 198]

In letters that same year to Herbrand $(1931, \# 2)^{1}$ and von Neumann, ${ }^{2}$ he defends the position that, for any correct formal system, there could be finitary proofs that escape it. On the face of it, his grounds seem to be that the notion of a finitary proof is not well-defined. Thus in letter \#3 (4.2.31) to Bernays, he writes

By the way, I don't think that one can rest content with the systems $\left[Z^{*}, Z^{* *}\right]$ as a satisfactory foundation of number theory (even

\footnotetext{
${ }^{1}$ I will cite Gödel's correspondence by giving the date and the number of the letter in Kurt Gödel: Collected Works, Volumes IV and V [Gödel, 2003a; Gödel, 2003b].

${ }^{2}$ In letter \# 3 (1931), von Neumann is disagreeing with Gödel's view on the formalizability of "intuitionism", by which he meant finitism. The letter to which he was replying has not been found.
} 
apart from their lack of deductive closure), and indeed, above all because in them the complicated and problematical concept "finitary proof" is assumed (in the statement of the rule for axioms) without having been made mathematically precise.

$Z^{*}$ and $Z^{* *}$ are systems defined by Hilbert's so-called $\omega$-rule and an extension of it proposed by Bernays, where the application of these rules makes essential reference to the notion of finitary proof.

In his earlier writings, Gödel does not say exactly what he believes Hilbert's finitism to be and, between 1931 and 1958, he had nothing really to say at all about finitism in his writings. But it is plausible to think that, when in 1858 he wrote "finitary mathematics is defined as the mathematics in which evidence rests on what is intuitive" (anschaulichen) [Gödel, 1958, p. 281], he was expressing what he had always taken to be Hilbert's conception. As far as I know, Hilbert's "Über das Unendliche" [Hilbert, 1926] is the only source in Hilbert's writings on finitism or proof theory that he ever cited, and surely his characterization of finitary mathematics accurately reflects what he would have read there.

But the view expressed in the Dialectica paper [Gödel, 1958, p. 28081] and essentially repeated at the end of his career in his English version (unpublished by him) is somewhat weaker than his earlier statements. In the latter, he still writes

Due to the lack of a precise definition of either concrete or abstract evidence there exists, today, no rigorous proof for the insufficiency (even for the consistency proof of number theory) of finitary mathematics. [1972, p. 273]

(He had just previously, in footnote $b$, introduced the aforementioned term "concrete intuition" to translate Kant's "Anschauung" and had written that "finitary mathematics is defined as the mathematics of concrete intuition", so there seems to be no doubt that "concrete evidence" refers to the evidence based on concrete intuition. This would seem to be ample evidence that Gödel is still expressing the view that finitism is the mathematics in which evidence rests on what is concretely intuitive.)

But there is this difference between his view in 1958 and later and the view that he expressed in 1931: He now goes on to state that in spite of the lack of a precise definition of what constitutes concrete or abstract evidence, the insufficiency of finitary mathematics for the proof of consistency of $P A$ 
"has been made abundantly clear by the examination of induction by $[\mathrm{sic}] \epsilon_{0}$ ". The argument as stated in [1972, p. 273] is that induction up to arbitrary ordinals $<\epsilon_{0}$ is not finitarily valid and that it could be proved finitarily if the consistency of $P A$ could. We will discuss this argument below, but for the moment I want only to point out that, although it does not contradict his earlier view that finitary mathematics is open-ended, it does contradict the view that his second incompleteness theorem does not close out the possibility of a finitary consistency proof of $P A$.

In this connection, though, one should note that, in the 1960's and 1970's, there was indeed some mind-changing in his correspondence with Bernays and in the revised version [Gödel, 1972] of the Dialectica paper over the question of whether or not $\epsilon_{0}$ is the least upper bound of the finitist ordinals. Although he concluded, in [Gödel, 1958; Gödel, 1972], that no valid argument had been given that it is the least upper bound, there are three references in his correspondence with Bernays to proofs that it is. One is an argument by Kreisel $[1960 ; 1965]$, which admits as finitist a reflection principle for which I can see no finitist justification. See [Gödel, 2003a, letter \# 40 (8/11/61)] and Tait $[1981 ; 2006]$. Gödel himself in $[1972$, p. 274, fn.4 and fn. $f]$ explicitly recognizes that this goes beyond finitary reasoning in Hilbert's sense. A second proof Gödel cites [2003a, letter \# 68b (7/25/69)] is mine [Tait, 1961], which proves in this connection only that recursion on $\omega^{\alpha}$ is reducible to nested recursion on $\omega \times \alpha$. The third [Gödel, 2003a, letter \#68b (7/25/69)] is Bernays' argument for induction up to $<\epsilon_{0}$ in the second edition of Grundlagen der Mathematik, Volume 2. But this argument is simply a nicer proof of (essentially) the result just mentioned from [Tait, 1961] and, again, does not yield a finitary proof of induction up to $<\epsilon_{0}$. (See [Tait, 2006, pp. 90-91] for a discussion of this.)

But none of these changes concern Gödel's conception of finitism as the mathematics whose evidence rests on concrete intuition. They only concern the possibility of analyzing this kind of intuition and placing bounds on it.

2. The fly in the otherwise smooth ointment of this story of Gödel's conception of finitism, however, is the set of notes for his lecture at Zilsel's in 1938 [Gödel, 1938a], in which he describes a system which he calls 'finitary (finite) number theory'. The lecture notes are quite rough, but it seems uncontroversial that the system he describes is primitive recursive arithmetic, 
$P R A$, which is a formalizable system. ${ }^{3}$ This fits neither his early view that finitism is open-ended nor the view that he later on entertained, that $\epsilon_{0}$ is the least upper bound of the finitist ordinals.

Before discussing this further, however, we need to distinguish two questions. One is: How did Gödel think the term "finitary" ("finit") should be applied? The other is: How did he understand Hilbert to be applying the term? (Two others are: How should the term be applied? And: How did Hilbert apply it? I gave my own answer to the first of these questions in [Tait, 1981]. I will briefly discuss the second here; but mainly I remain agnostic about whether Gödel correctly understood Hilbert.) I believe that the evidence is overwhelming that, aside from his use of the term "finitary" in the Zilsel lecture and in one other case, in all of the instances cited above of his use of the term, it referred to what he took Hilbert to mean by it: In almost every case, either there is a direct reference to Hilbert or the context of the remark is a discussion of Hilbert's finitism.

That, in the Zilsel lecture notes, he has something else in mind is clear already from the fact that he is describing a hierarchy of systems which he calls 'finitary.' These include not only finitary number theory (PRA), which he calls the lowest level of the hierarchy, but systems which extend beyond this, involving functions of higher type, or the logical operations (which he calls the 'modal-logical route'), or transfinite induction [Gödel, 1938a, pp.93-4]. The latter systems clearly go beyond the mathematics of concrete intuition; and so it has to be concluded that he was not using the term 'finitary' ('finit') here to refer to Hilbert's finitism.

Incidentally, referring to finitary number theory, he writes "I believe that Hilbert wanted to carry out the proof [[of consistency]] with this" (p. 93) This may seem to hint at the view that Hilbert identified finitism with PRA, but that is not what he is saying. He is repeating what is written in his notes [Gödel, *1933o, p. 25] for his lecture at a meeting in 1933 of the MAA (joint with the AMS). There he wrote that the methods used in attempts to prove consistency "by Hilbert and his disciples" are all expressible in a system

\footnotetext{
${ }^{3}$ These lecture notes, together with those for his lecture in 1933 at a joint meeting of the AMA and the AMS, play a central role in my discussion. In neither case does the introductory note in [Gödel, 1995] indicate any other source of information about the actual content of the lecture. Given the nature of lecture notes in general and in particulardespite the heroic efforts of the editors to clarify their content - the exceedingly sketchy character of the Zilsel lecture notes, special care is needed in assessing their intended meaning.
} 
that he called 'system $A$ ', but which by 1938 he could identify with $P R A$. (We will discuss the connection between $P R A$ and the system $A$ presently.) Literally, Gödel is mistaken about this: As Richard Zach has noted [Zach, 2003], Hilbert had approved as finitist Ackermann's use of induction up to $\omega^{\omega^{\omega}}$ in his dissertation [Ackermann, 1924]. In fact, though, the only instance of transfinite induction for which Ackermann gave any justification in his paper was induction up to $\omega^{2}$ and his argument was essentially just the reduction of this to primitive recursion with substitution in the parameters (which of course reduces to primitive recursion). It seems quite possible that Hilbert mistakenly believed that one could with some further complication likewise derive the stronger cases of induction in PRA. (I discuss this further in [Tait, 2005b, Appendix to Chapters 1 and 2].) In any case, there is no reason to believe that Gödel should have been aware of this: In Über das Unendliche [Hilbert, 1926], the Ackermann function (induction up to $\omega^{\omega}$ ) is not taken to be finitist. (See the discussion of this in [Tait, 1981].)

In the notes for the 1933 lecture, he described the hierarchy of systems analogous to the 1938 one as 'constructive', rather than as finitary. The explanation of why Gödel applied the term "finit" to the systems in this hierarchy in 1938 is probably given in the notes for his lecture at Yale [Gödel, *1941] on the Dialectica interpretation, where he does so as well. He writes

Let me call a system strictly constructive or finitistic if it satisfies these three requirements (relations and functions decidable, respectively, calculable, no existential quantifiers at all, and no propositional operations applied to universal propositions). I don't know if the name "finitistic" is very well chosen, but there is certainly a close relationship between these systems and what Hilbert called the "finite Einstellung".

The conditions that he places on a finitary system in the Zilsel lecture notes differ slightly from those in the Yale lecture notes, but not significantly.

3. There are hints in later writings that, independently of what Hilbert thought, "finitism" might have a more extensive meaning for Gödel simply because the term "intuition" does. Just prior to his statement that "finitary mathematics is defined as the mathematics in which evidence rests on what is what is intuitive" in [1958], he sets the stage for this by specifying that he 
is speaking about "what is, in Hilbert's sense, finitary mathematics". (The italics are mine.) The footnote $b$ in the 1972 version is even more suggestive:

"Concrete intuition", "concretely intuitive" are used as translations of "Anschauung", "anschaulich". ...'What Hilbert means by "Anschauung" is substantially Kant's space-time intuition confined, however, to configurations of a finite number of discrete objects. Note that it is Hilbert's insistence on concrete knowledge that makes finitary mathematics so surprisingly weak and excludes many things that are just as incontrovertibly evident to everybody as finitary number theory. E.g., while any primitive recursive definition is finitary, the general principle of primitive recursive definition is not a finitary proposition, because it contains the abstract concept of function. There is nothing in the term "finitary" which would suggest a restriction to concrete knowledge. Only Hilbert's special interpretation of it introduces this restriction.

Gödel simply doesn't see the 'finite' in 'finitary': He sees 'concrete intuition' instead, and he questions Hilbert's restriction to the concrete. ${ }^{4}$ By "abstract" he refers to concepts

which do not have as their content properties or relations of concrete objects ... but rather of thought structures or thought contents (e.g., proofs, meaningful propositions, and so on), where in the proofs of propositions about these mental objects insights are needed which are not derived from a reflection upon the combinatorial (space-time) properties of the symbols representing them, but rather upon a reflection upon the meanings involved. ([Gödel, 1972, pp.272-3] Cf.[Gödel, 1958, p. 280]

I believe it would be very hard to defend his reference to such higher-order objects, where are also to include functions of finite types over the numbers, as "mental". They are, after all, in the public domain, i.e. objective: we

\footnotetext{
${ }^{4}$ [Bernays, 1930-31] argues precisely for the view that the 'finite' in finitism is a corollary of, let us say, concrete intuition. But I do not know whether Gödel ever read that paper. It is striking that in Gödel's correspondence with Bernays, neither ever refers to the latter's quite obvious involvement in the 1920's in constructing the finitist ideology. It is as if Bernays were merely a witness to Hilbert's development of it.
} 
define them and reason about them together. If we disagree about the truth of an numerical equation, we believe that one of us is right and the other wrong, not that you have your successor function and I have mine. If we restrict the higher-order objects - proofs of arithmetic sentences, functions of finite type over $\mathbb{N}$, etc.- to ones that are computable, then they can be represented by Turing machines and so, in this sense, are finite. But then, on the face of it, our reasoning about them qua finite objects (Turing machines) involves nontrivial arithmetic: For example, the definition of a computable object of type $A$ has roughly the same logical complexity as $A$ itself (where $A$ may be a finite type over the natural numbers or a formula of $P A$ ).

Gödel seems to have had in mind a kind of evidence that one might say rests on abstract intuition, that goes beyond concrete intuition, but remains logic free. This seems to be the idea that he was trying to work out-but never succeeded - in the Dialectica paper and its revision (which he never released for publication). But it is a different conception of intuition from the kind of intuition he speaks of in [Gödel, *1961/?; Gödel, 1964], where intuition is invoked as a source of new axioms in set theory. Charles Parsons [1995, pp. 57-58] makes the distinction between the concrete intuition of Hilbert's finitism and intuition in the sense that it is used in [Gödel, 1964; Gödel, *1961/?] and discusses the latter in some detail. I am suggesting that for Gödel there was another conception of intuition, to which I am referring as 'abstract intuition', which would play the same foundational role as concrete intuition. For the purpose of consistency proofs it was essential, on pain of circularity, that the methods used to prove consistency - finitism or proposed extensions of it-rest on a different, non-axiomatic foundation from the axiomatic theories whose consistency is to be proved.

Anticipating $\S 4$ below, if we understand that what is given with 'concrete intuition' is finite iteration, as an operation that can be applied to operations on finitary domains (i.e. domains which can be represented by the domain of natural numbers), then a natural extension to 'abstract intuition' arises simply by admitting iteration to be applied to operations on non-finitary domains, such as the domain of numerical functions, the domain of function on these, etc. On this basis, one would then have a foundation in abstract intuition for Gödel's theory of primitive recursive functions of finite type, although not the one Gödel was after. That is because of his finiteness requirement: He wanted to restrict the domain of functions of type $n+1$ to the hereditarily computable ones, i.e. to those which are computable applied to computable functions of type $n$. And he required that this notion 
of 'computable function of type $n$ ' be understood in some way that is logicfree - and that is why he failed. See [Tait, 2005a] for a fuller discussion of this. We note here that, again giving up the finiteness requirement, this same 'abstract intuition' also provides a basis for intuitionistic first-order number theory.)

But this 'abstract intuition', too, is very different from that discussed in [Gödel, *1961/?]. In set theory the finite iteration 'given in intuition' is analyzed away, à la Frege/Dedekind.

4. In [*1961/?], Gödel related intuition as a source of new axioms to Kant's conception of intuition and its role in mathematics. In this and the next section, I want to comment on the relation between Gödel's conception(s) of intuition and Kant's. Gödel's conception seems to correspond well with Leibniz's notion of intuition: a direct insight into truth, which is the starting point of proof - although, as Parsons points out [1995, p. 61], for Gödel the insight need not be infallible. For both Leibniz and Gödel, intuition is propositional: To use Parsons' term [1995, p. 58], it is intuition that. ${ }^{5}$ Here is what Gödel writes in [*1961/?]:

I would like to point out that this intuitive grasping of ever newer axioms that are logically independent from the earlier ones, which is necessary for the solvability of all problems even within a very limited domain, agrees in principle with the Kantian conception of mathematics. The relevant utterances by Kant are, it is true, incorrect if taken literally, since Kant asserts that in the derivation of geometrical theorems we always need new geometrical intuitions, and that therefore a purely logical derivation from a finite number of axioms is impossible. [Gödel, *1961/?, p. 10]

This is a mistake, and it is this incorrect understanding of Kant that supports Gödel's view that reasoning based upon concrete intuition is open-ended. Indeed, as I will argue below, on the most plausible reading, a development of Kant's philosophy of arithmetic leads precisely to $P R A$.

However much Kant may have on occasion used the term "intuition" ( $A n$ schauung") in the propositional sense, it is a fundament of his philosophy

\footnotetext{
${ }^{5}$ It is true that intuition of may be suggested by "in this kind of perception, i.e., in mathematical intuition" in [1964, p. 271]. But, as I argued in [Tait, 1986, fn. 3], the context makes it clear that it is propositional knowledge - namely axioms - that the intuition is to be yielding.
} 
to distinguish sensibility, the faculty of intuition, from understanding, the faculty of concepts, and there is no doubt but that, in this context, intuition is intuition of, the unique immediate mode of our acquaintance with objects: All objects are represented in sensible intuition. Abstracted from its empirical content the intuition is just space (pure outer intuition) and time (pure inner intuition). He also speaks of (sensible) intuitions of objects to refer to their representations in intuition. But an intuition by itself is not knowledge: The latter requires recognizing that an object represented in intuition falls under a certain concept or that one concept entails another. $A$ priori knowledge of the latter sort, that all $S$ are $P$, may be analytic, namely when $P$ is contained in $S$. But, although the truths of mathematics can be known a priori, they are not in general analytic. When they are not analytic, the connection between subject and predicate is mediated by construction. ${ }^{6}$ The demonstration of the proposition begins with the 'construction of the concept' $S$. Thus, to take one of Kant's examples, to demonstrate that the interior angles of a triangle equal two right angles, we construct the concept 'triangle', construct some auxiliary lines, and then compute the equality of the sums of two sets of angles, using the Postulate "All right angles are equal" and the Common Notions "Equals added to (subtracted from) equals are equal". The construction of a concept is according to a rule, which Kant calls the schema of the concept. In the case of geometric concepts these are or at least include the rules of construction given by Euclid's 'to construct' postulates, Postulates 1-3 and 5. Of course, these rules are rules to construct objects from given objects. For example, given three points $A, B, C$, we can

\footnotetext{
${ }^{6}$ It has frequently been asserted that at least some of the explanation for Kant's theory of sensibility and the distinction between demonstration in mathematics and discursive reasoning lies in the poverty of logic in his time: Construction and computation could not be expressed as logical processes. But another, complementary, way to understand him is to see that he was expressing and attempting to underpin what was in his day a commonly held conception of mathematics, as primarily involving computation and construction. So long as $\epsilon-\delta$-arguments could be hidden behind infinitesimals, this view of mathematics could prevail, at least if not too closely scrutinized. It was precisely the evolution of mathematics in the nineteenth century, which forced complex $\epsilon-\delta$-arguments out into the open, that led to the development of logic - in particular, quantification theory-in which computation and construction could be expressed. (This latter point was made in a talk by William Ewald in a workshop on Hilbert's program in 1995 at Carnegie-Mellon University and the University of Pittsburgh.) It of course also led to a more structural conception of mathematics itself, in which computation and construction yielded to logic and existence axioms and proofs. (See [Stein, 1988].)
} 
construct the three lines joining them and thereby, assuming that they are non-collinear, construct the triangle $A B C$.

Kant recognized that we cannot be speaking of empirical construction here, although he doesn't explicitly give as a reason that empirical objects fail to satisfy the assumptions of geometry. Rather he was concerned with the fact that any empirical figure would be too special to satisfy the requirement that the demonstration apply generally: For example, the empirically constructed triangle is either a right triangle or not - at least if we ignore the fact just mentioned, that they are not really triangles at all. If right, then why does the demonstration apply to those triangles which are not? And conversely. ${ }^{7}$ Kant's solution to this problem is that we construct the figure in imagination: Thus, we imagine three non-colinear points $X, Y, Z$ and construct from these the triangle $X Y Z$ and whatever auxiliary objects that are needed. These points are indeterminate, in the sense that their only properties are those we put into them - in other words, those implied by the original assumption that they are non-colinear. In general, we are able to construct figures in imagination that contain only the properties that we put into them. The construction of the triangle therefore serves as a template for demonstrating the theorem for any three empirical non-colinear points.

There is no general agreement about how to understand this construction in imagination. Are we to understand that an object is really being constructed in imagination - that the construction-of-a-triangle-from-the-givenpoints in imagination is the construction of an object from given objects, but from ones which are in the relevant sense 'indeterminate'; ${ }^{8}$ or are we to understand that no objects are given and no object is being constructed at all, but that it is the total construction itself, regarded as a function that can be applied to any system $A, B, C$ of empirical points, which is the object of pure geometry? On this latter view, developed in the writings of Michael Friedman (see especially [Friedman, 1992; Friedman, 2008]), there are no objects of pure intuition: rather pure intuition, i.e. space, is simply the context of the constructions, giving the rules of construction meaning. ${ }^{9}$

\footnotetext{
${ }^{7}$ Interpreting Kant to hold that we construct the triangle in pure intuition or that it is itself a 'pure intuition' presents the same difficulty: if its a triangle, then it is either a right triangle or it is not.

${ }^{8} \mathrm{I}$ think it is enlightening here to think of the indeterminates $X_{1}, \ldots, X_{n}$ in the polynomial extension $R\left[X_{1}, \ldots, X_{n}\right]$ of a ring $R$.

${ }^{9} \mathrm{I}$ suggest the view that it is the latter idea that Kant was aiming for, but that a sufficiently general notion of function was unavailable to him; and so in the construction
} 
On this view, geometry qua mathematics is really about these constructions, about the functions. The theorems of geometry (roughly) apply to empirical figures because our representation/construction of these figure in empirical intuition is in accordance with these self-same rules.

But on neither view is intuition intuition that.

So, on the basis of this, what can be made of Gödel's assertion "Kant asserts that in the derivation of geometrical theorems we always need new geometrical intuitions", quoted above? Presumably, the "new geometrical intuition' needed for the theorem that all $S$ are $P$ is in the construction of the concept $S$ or in the auxiliary constructions. But Kant is quite clear that all of these constructions are according to the schemata for the concepts in question. And there is nothing in Kant's writings to indicate a belief that any new rules of construction are needed, much less one for the demonstration of each new geometric theorem. Yet it is these rules of construction, that correspond, via the transformation from "To construct an $x$ such that ..." to "There exists an x such that ...", to axioms. So I think that Gödel's conception of intuition in [Gödel, 1964; Gödel, *1961/?] has little to do with Kant's notion of intuition.

5. Kant had rather less to say about arithmetic than he did about geometry. ${ }^{10}$ Corresponding to the concepts of triangle, line, etc., in arithmetic there is the concept magnitude (Grösse and, in parenthesis, quantitatis. ${ }^{11}$ The schema according to which we construct this concept is number. About this he writes

The schema is in itself always a product of imagination. Since, however, the synthesis of imagination aims at no special intuition, but only at unity in the determination of sensibility, the schema has to be distinguished from the image. If five points be

of the triangle in imagination, for example, there are imagined points $X, Y, Z$ which play the role of indeterminates - non-colinear, but otherwise indeterminate in their relations with one another-in the construction.

${ }^{10}$ So far as I can recall, the single example he discusses in $C P R$ of a synthetic arithmetical proposition is $7+5=12(B 15-16)$, which he states is synthetic because being 12 is not contained in the concept of being $7+5$ : Only the concept of being some number is contained in it. But I don't understand that: How does the concept of a totality consisting precisely of seven things and five other things contain the concept of having a (finite) number? It would seem that that too would be a synthetic judgment. See footnote 12 below.)

${ }^{11}$ This refers to magnitude in the sense that two distinct geometric figures can have the same magnitude. Kant distinguishes this from the (Greek) concept of magnitude according to which the geometric objects themselves are the magnitudes. 
set alongside one another, thus, ... I have an image of the number five. But if, on the other hand, I think only a number in general, whether it be five or a hundred, this thought is rather the representation of a method whereby a multiplicity, for instance a thousand, may be represented in an image in conformity with a certain concept, than the image itself. ... . This representation of a universal procedure of imagination in providing an image for a concept, I entitle the schema of this concept. (B179)

He continues at $B 182$ :

[T] he pure schema of [the concept of] magnitude ... is a representation which comprises the successive addition of homogeneous units. Number is therefore simply the unity of the synthesis of the manifold of a homogeneous intuition in general, a unity due to my generating time itself in the apprehension of the intuition. ${ }^{12}$

So the schema of the concept of magnitude, i. e. number, is finite iteration, which he conceived as always taking place in time, as in counting - that is why arithmetic is associated with inner intuition. In taking number to be the schema of magnitude, he is recognizing the fundamental role of counting in measurement - comparing two lines, for example, with respect to how many units they contain. Thus:

No one can define the concept of magnitude in general except by something like this: That it is the determination of a thing through which it can be thought how many units are posited in it Only this how-many-times is grounded on successive repetition, thus on time and the synthesis of the homogeneous in it. (B300, italics mine)

\footnotetext{
${ }^{12}$ The reader who finds all of this perfectly clear might consider taking up Kant scholarship - and, as an initial exercise, answering the following question: It would seem that Kant's assertion that the concept of consisting of precisely seven things and five others includes the concept of having a number must be understood now to mean that it includes the concept of being a quantity. But the concept of quantity applies only via the schema, number, which consists in counting it out. So how do we know that the totality can be counted out without either counting it out (and so knowing that the sum is 12) or invoking the principle that $7+V$ is a quantity for arbitrary (indeterminate) quantity $V$ ? But in either case, the knowledge would seem to be synthetic, involving construction in accordance with the schema number.
} 
If we follow the model given by Kant's geometric examples, we may construct in productive imagination the quantity (i.e. finite iteration) $f\left(V_{1}, \ldots, V_{n}\right)$ from 0 (the null iteration), the construction +1 (of iterating once more), and the indeterminate quantities (finite iterations) $V_{1}, \ldots, V_{n}$. Here the indeterminate finite iterations $V_{1}, \ldots, V_{n}$ correspond to the indeterminate points in the case of geometry. A proof of the general arithmetical proposition $f\left(V_{1}, \ldots, V_{n}\right)=0$ would also be a construction in a finite number of steps. But, on pain of failing to found any non-trivial arithmetic, a single step in the construction either of $f\left(V_{1}, \ldots, V_{n}\right)$ or of the proof of $f\left(V_{1}, \ldots, V_{n}\right)=0$ may consist of applying one of the finite iterations $V_{1}, \ldots, V_{n}$ itself to iterate a construction obtained at earlier steps. For example, we construct the number $7+V$ as $(+1)^{V}(7)$. Again, suppose we have constructed a proof $p$ of $f(0)=0$ and a proof $\phi(V)$ of $f(V)=0 \Longrightarrow f(V+1)=0 . \phi(V)$ is to be understood as a computation of $f(V+1)=0$ from an arbitrary computation of $f(V)=0$. Then a proof $\psi(V)$ of $f(V)=0$ is defined by

$$
\psi(0)=p \quad \psi(V+1)=\phi(V) \psi(V)
$$

Here we understand the right-hand side of the second equation to be the result of applying $\phi(V)$ to the particular computation $\psi(V)$. This definition is not a pure iteration; but it is not difficult to reduce it to one. (See [Tait, 2005a].)

Thus, as in the case of geometry, the role of intuition is not that of a source of truth ('intuitive truths'). Rather it is this: We may imagine an arbitrary quantity $V$, a finite iteration, and, in pure inner intuition (i.e. time) construct a quantity $f(V)$ or construct a proof of a proposition $f(V)=0$ from it.

This conception can be developed to provide a foundation for definition of functions of a numerical variable and proof of equations by mathematical induction (see [Tait, 1981] and [Tait, 2005a, §1] for details) that is alternative to the axiomatic foundation involving second-order logic in [Dedekind, 1888]. Dedekind himself was quite explicit about wanting to eliminate 'inner intuition' from the foundations of arithmetic (see p. iv of the preface to the first edition); but when Hilbert began to attack the problem of proving the consistency of axiomatic theories, he or, better, he and Bernays came to realize that, on pain of circularity, one needs a different kind of foundation for mathematics of the consistency proofs themselves, and returned (for this) in 1922 to Kant's - or at least what Hilbert took to be Kant's - foundation. ${ }^{13}$

\footnotetext{
${ }^{13}$ In [1931, p. 8], Hilbert mentioned Kronecker's conception of mathematics in this con-
} 
If iteration is restricted to operations on domains of finite objects, say the domain, for each $n$, of $n$-tuples of natural numbers or (as in the above example) a domain of computations, then this conception leads to the system $P R A$. As I mentioned $\S 2$, I have argued, independently of what Kant, Hilbert and Bernays, or Gödel believed, that this is what the term "finitism" should mean.

Of course, $P R A$ goes well beyond what one finds in Kant's writings. I only want to argue that it embodies a logic-free conception of arithmetic based on iteration that naturally derives from Kant, but in which iteration is freed from the temporal character it has in Kant's theory. This 'Kantian' conception of arithmetic is in rough agreement with the intuitionism of Poincaré and Weyl. For the former the principle of iteration is given in intuition and is the one synthetic a priori truth of mathematics (see [Poincaré, 1900]) and for the latter, at least in his intuitionistic phase, it is the basis of all arithmetic, the one principle that need not and indeed cannot be proved. See [Weyl, 1921], the end of Part II §1, "The Basic Ideas", and the first paragraph of $\S 2 \mathrm{a}$ ("Functio Discreta"), and also [Weyl, 1949, p. 33]. Weyl had already rejected Dedekind's foundation of arithmetic on grounds of its impredicativity in Das Kontinuum [1918].

6. As was mentioned above, Gödel's only reference to Hilbert's writings concerning finitism is the one cited above, to "Über das Unendliche". Here is the relevant part of what Hilbert wrote:

Kant already taught - and indeed it is part and parcel of his doctrine - that mathematics has at its disposal a content secured independently of all logic and hence can never be provided with a foundation by means of logic alone;... Rather, as a condition for the use of logical inferences and the performance of logical operations, something must already be given to our faculty of representation, certain extralogical concrete objects that are intuitively present as immediate experience prior to all thought. If logical inference is to be reliable, it must be possible to survey these objects completely in all their parts, and the fact that they occur, that they differ from one another, and that they follow each other, or are concatenated, is immediately given intuitively, to-

nection in opposition to Dedekind's foundations and expressed the view that Kronecker's conception "essentially coincides with our finite mode of thought." 
gether with the objects, as something that neither can be reduced to anything else nor requires reduction. This is the basic philosophical position that I consider requisite for mathematics and, in general, for all scientific thinking, understanding, and communication. And in mathematics, in particular, what we consider is the concrete signs themselves, whose shape, according to the conception we have adopted, is immediately clear and recognizable.

The concrete objects Hilbert had in mind are the words over a finite alphabet, since he was interested in reasoning about the expressions in formal axiomatic systems; but of course it suffices for us to think only about the case of reasoning in arithmetic, which concerns the words

$$
|| \cdots \mid
$$

over the single letter $\mid$. In the passage quoted above, Kant refers to these words - or at least to particular physical instantiations of them-as images of numbers. From a Kantian point of view, it seems fair enough to represent particular quantities by these words. But the words are dead: What are we supposed to do with them? There is no trace in the passage just quoted from Hilbert of the central role of iteration, of the Kantian conception of iteration as the schema for constructing quantities. In fact Hilbert gives no account of how we are to reason about these words in general, except for the negative requirement that the reasoning be logic-free. This is not only true of the passage in question: The criticism extends as far as I know to all of the writings of Hilbert and Bernays on finitism. We are given examples of finitist reasoning and examples of non-finitst reasoning, but we are not told what finitist reasoning is. For example, the rule of definition and proof by mathematical induction emerges as a finitist principle, on the grounds that the words over | are built up by finitely iterating the operation of adding another |; but it is in no way marked out, as it was by Weyl, as the principle of finitist reasoning. It is not explicitly excluded by them that logic-free principles of transfinite induction up to some $\alpha$ (represented by an ordering of the natural numbers) can have equal claim to being finitist, even though they might not be derived from mathematical induction. But on what grounds does one distinguish between those principles that are finitist and those that are not? ${ }^{14}$ I believe that no clear answer is given to this question by Hilbert and

\footnotetext{
${ }^{14}$ A minimalist reading of the above passage from "Über das Unendliche" has led Parsons
} 
Bernays. In particular, although the issue has been debated on the battlefield of $\S 7$ of Grundlagen der Mathematik, Volume 1 [Hilbert and Bernays, 1934] (see [Zach, 1998], [Tait, 2002] and [Tait, 2005b, Appendix]), the question of whether Hilbert and Bernays, in the period 1922 until 1931, when the quest for consistency proofs was framed in terms of finitism and before the need to extend that framework became apparent, regarded reducibility to $P R A$ as the essential mark of finitist definition and proof is not, in my opinion, definitively resolved.

On the other hand, the absence of a clear statement of that criterion could lead one to the presumption that they did not accept it. There are some other bits of information that tend towards that conclusion. One concerns what has been misnamed 'Hilbert's $\omega$-rule' in his [1931]. This asserts that, if the quantifier-free formula $A(n)$ is true for all numbers $n$, then $\forall x A(x)$ may be taken as an axiom. "True" here has to mean true on finitist grounds and, moreover, Hilbert refers to this principle as being finitary. One can reasonably conclude that the principle can be restated as saying that $\forall x A(x)$ may be taken as an axiom if $A(V)$ is finitistically valid, for the arbitrary (indeterminate) number $V$. But if Hilbert intended finitism to be limited to what can be defined and proved in PRA, his ' $\omega$-rule' amounts simply to adding $P R A$ to the formalism. Likewise, in a letter [Gödel, 2003a, letter \#2 (1931)] to Gödel, written in 1931, Bernays suggests that perhaps the incompleteness theorems do not apply to the result of adding this rule to first-order arithmetic. Certainly if he were identifying what is finitistically provable with

to the conclusion that even primitive recursion, in particular, exponentiation, cannot be founded on intuition [Parsons, 1998, p. 265]. He believes that not even the elaboration of the finitist point of view in [Bernays, 1930-31] avoids this conclusion (p. 263); but that is not clear to me. If one takes as the basis of finitism not 'intuitions' of inert symbol complexes, but 'processes', to use Bernays' term, then exponentiation and, indeed, all of the primitive recursive functions become accessible. It remained only for Bernays to be more specific about the processes in question and explain why, or better, in what sense they are founded on intuition. He didn't do that. But I don't see that this justifies Parson's position: It isn't that we are told exactly how to reason about the dead symbols and what the limits of such reasoning are, and that what we are told does not support exponentiation. Aside from examples, we are told nothing about how to reason concerning them, except that it should be logic-free. It is true that a certain sketch is given of how to understand addition and multiplication finitistically, using the operations of concatenation and replacement (of each occurrence of $\mid$ in a word by a given word), where there is no reasonable extension of this sketch to exponentiation. But these constructions are themselves just examples. The general principle of primitive recursion is another example that is given. 
what is provable in $P R A$, he would have known that this suggestion is false. More generally, in all of his published correspondence with Gödel, Bernays nowhere suggests that $P R A$ was understood to be the criterion for finitist meaningfulness and validity. Indeed, in letter \#68b (7/25/69) to Bernays, Gödel states that "nested recursions are not finitary in Hilbert's sense (i.e. not intuitive)." In his reply, letter \#69 (1/7/70), Bernays remarks that he feels that manifold nested recursions are finitary in the same sense as primitive recursions. But Bernays was then eighty-two years old, and (although I grow increasingly reluctant to do so) it seems reasonable to somewhat discount what he said then as evidence for his or Hilbert's views in the 1920's. In fact, the argument he sketches involves iteration applied to an operation on the non-finitist domain of numerical functions.(See [Tait, 2002, pp. 415-416].) The question remains whether in the 1920's Hilbert would have accepted a definition or proof as finitist that he knew could not be reduced to PRA.

Of course, there is an oft-stated view, perhaps suggested first by Kreisel (see [Kreisel, 1958]), with which I have considerable sympathy, that Hilbert's attitude towards what counts as finitist was pragmatic: Find the consistency proof and then see what has been used.

7. Let me turn to another puzzle in connection with Gödel's conception of finitism, concerning the system $A$ described in the lecture notes [Gödel, *1933o] for his lecture at a meeting of the $M A A$ (held jointly with the $A M S$ ). It should be noted that Gödel does not use the term "finitism" or "finitary" in the 1933 lecture notes at all, and in particular, as we have noted, he refers to this hierarchy as a hierarchy of 'constructive' systems. But nevertheless, there is a puzzle about the relationship between finitary number theory $(P R A)$ in the 1938 lecture notes and the system $A$ : Both are described as being at the lowest level of the hierarchy. ${ }^{15}$

$A$ is a free-variable system based on the principle of both definition and proof by complete induction. It differs from the finitary number theory of the 1938 lecture, however, in that it admits, besides the domain of natural numbers, other totalities for which "we can give a finite procedure for generating all of their elements" [Gödel, *1933o, p.23]. Since complete induction is to be valid when applied to the generating procedure in each case [ibid, p. 24], clearly there must be a unique generation for each object. Thus,

\footnotetext{
${ }^{15}$ I have discussed this in some detail also in [2006, §9, pp. 98-105].
} 
the totalities in question can be represented by certain 'finitely generated' totalities of words over some alphabet (finite or itself a finitely generated totality) - which, in view of the fact that $A$ is to be a foundation for proof theory, concerned with syntactical systems of the sort studied in the Hilbert school, is undoubtedly what Gödel had in mind. The admission of such totalities, other than the totality of numbers, is the sole difference between the system $A$ and the finitary number theory of the Zilsel lecture notes.

Why this difference between the system $A$ in 1933 and the system of finitary number theory of 1938? A related question is why in the notes for the 1933 lecture he wrote that

there are reasons for believing that [any intuitionistic proof complying with the requirements of system $A$ ] can easily be expressed in the system of classical analysis and even in the system of classical arithmetic.

The answer, I think, lies in the fact that in 1933 he felt that what counted as a 'finite procedure', i.e. an effective procedure, was not convincingly analyzed, so that it was not fully established that all 'finitely generated' totalities of words could be, via Gödel-numbering, expressed in arithmetic. This is the doubt that he expressed one year later in footnote 3 of his lectures in Princeton on incompleteness [Gödel, 1934, p. 3, fn. 3].

On the other hand, as he suggested in that footnote, there was some ground for believing in the analysis of effective procedure in terms of general recursiveness, so that the totalities generated by finite procedures are all representable by recursively enumerable sets of numbers, i.e. by formulas $\exists y \phi(x, y)$ where $\phi$ is primitive recursive. Thus the totality of natural numbers is the only finitely generated totality required. Since primitive recursion can be derived in second-order number theory (as was already proved in effect by Dedekind) and even first-order number theory (as Gdel himself proved in [1931]), it follows that there was indeed a reason for believing that proofs in accord with the system A could always be expressed in classical analysis and even in classical arithmetic.

In some notes [Gödel, *193?], evidently for a lecture sometime in the 1930's, Gödel expressed his belief that the "gap" between the informal notion of computability and a precise mathematical definition "has been filled by Herbrand, Church and Turing." Certainly he is referring to the papers of Church and Turing in 1936. If we assume that he had that belief when he wrote his notes for the 1938 lecture at Zilsel's, this will explain why, in those 
notes, he restricted the lowest level of finitary mathematics to $P R A$, i.e., to the case of system $A$ in which the only totality involved for which "we can give a finite procedure for generating all of [its] elements" is the totality of natural numbers. ${ }^{16}$

8. As I mentioned earlier, the real change in Gödel's view of finitism was later, first visible in the Dialectica paper, [1958]. It is there that he asserts that induction on $\epsilon_{0}$ cannot be proved finitarily: I quote the slightly expanded passage in the 1972 version:

Recursion for $\epsilon_{0}$ could be proved finitarily if the consistency of number theory could. On the other hand the validity of this recursion can certainly not be made immediately evident, as is possible for example in the case of $\omega^{2}$. That is to say, one cannot grasp at one glance the various structural possibilities which exist for decreasing sequences, and there exists, therefore, no immediate concrete knowledge of the termination of every such sequence. But furthermore such concrete knowledge (in Hilbert's sense) cannot be realized either by stepwise transition from smaller to larger ordinal numbers, because the concretely evident steps, such as $\alpha \rightarrow \alpha^{2}$, are so small that they would have to be repeated $\epsilon_{0}$ times in order to reach $\epsilon_{0}$. [1972, p. 273]

I don't understand the first statement of this quote. Recursion up to $\alpha<\epsilon_{0}$ can be formalized by the formula "If $f(0)=a$, then there is a number $k$ such that $f(k+1) \nprec f(k)$ ", where $f$ is a free numerical function variable, $\prec$ is the standard ordering of all the natural numbers of order type $\epsilon_{0}$, and $a$ is the number representing $\alpha$ in that ordering. Indeed, as stated in [Gödel, 1958, pp. 281], Gentzen [1943] proved that, for each $\alpha<\epsilon_{0}$, this formula is deducible in $P A$. But why does the consistency of $P A$ finitarily imply these statements? Gentzen's own consistency proof, in the form that for every deduction in $P A$, every one of his reduction sequences terminates with a sequent $\emptyset \vdash A$, where $A$ is a true atomic sentence, does indeed imply this. (Roughly, the ordering up to $\alpha$ is embedded in the tree of reductions corresponding to the deduction

\footnotetext{
${ }^{16}$ The history of Gödel's reaction to Turing's analysis is somewhat more complex than I represented it to be in [2006] and in an earlier version of this paper. I have Wilfried Sieg to thank for pointing this out to me so that I avoided error (at least about this). As to the precise relationship between Gödel and Turing on computability and the issues involved, the reader should consult [Sieg, 2006].
} 
of recursion up to $\alpha$.) But I don't see why an arbitrary finitary consistency proof for $P A$ should translate into Gentzen's.

Nevertheless, the argument that recursion on ordinals $<\epsilon_{0}$ is not finitarily provable is interesting. In letter \#61(1/24/67) to Bernays Gödel wrote

I am now convinced that $\epsilon_{0}$ is a bound on Hilbert's finitism, not merely in practice but in principle, and that it will also be possible to prove that convincingly.

The argument in the 1972 paper is a considerable expansion of his remarks in 1957; and so it is reasonable to assume that it contains the analysis which led to the conviction. The basic notion is that of "grasping at one glance the various structural possibilities which exist for decreasing sequences" from a countable ordinal $\alpha$-lets just call this grasping $\alpha$. We can assume that the descending sequences are in fact paths through $\alpha$ conceived as an upsidedown connected tree: 0 is the null tree, $\alpha+1$ is the tree obtained by adding one node above the tree $\alpha$, and $\lim _{n<\omega} \alpha_{n}$ is the tree whose immediate subtrees are the $\alpha_{n}$. (Of course, this distinguishes ordinals that are classically identifiede.g. $\omega=\lim _{n} n$ with $\lim _{n<\omega} 2 n$. But that is of no consequence for the discussion.)

Surely 1 is graspable and, if $\alpha$ and $\beta$ are graspable, then so is $\alpha+\beta$, since its paths are just the concatenation of a path through $\beta$ followed by one through $\alpha$. Indeed, if $\alpha_{1}, \ldots, \alpha_{k}$ are graspable, then so is $\alpha_{1}+\cdots+\alpha_{k}$. Thus, when $\beta$ is graspable, then so is $\beta \times n$ for any fixed $n$. But we cannot explicitly 'grasp in one glance' an infinite number of things; and so if $\alpha$ is infinite and we grasp at one glance all the structural possibilities of paths through $\alpha$, then obviously the glance has to contain some schematic elements-some "...", representing the result of an arbitrary finitely repeated concatenation of paths through some given graspable ordinals. Thus, we can represent $\omega$ as the arbitrary finitely repeated concatenation of paths through 1: $\alpha+\omega$ can be represented by $\alpha \cdots_{1}$. I don't want to fully explore the point now, but given that we are speaking of 'immediate concrete knowledge' here, I don't see what candidates for an $\cdots$ are available other than than $\cdots_{\beta}$ for some graspable $\beta$, i.e. representing arbitrary concatenations of paths through $\beta$. Thus, if we admit this principle for constructing graspable ordinals, then $\omega^{0}=1$ and $\omega^{n+1}=0 \cdots \omega^{n}$ shows that $\omega^{n}$ is graspable for each particular $n<\omega$. But, if $\beta<\omega^{\omega}$, i.e. $\beta<\omega^{n}$ for some $n$, then $0 \cdots_{\beta}=\beta \times \omega<\omega^{\omega}$; and so on this analysis, $\omega^{\omega}$ (the ordinal of $P R A$ ) is not graspable. ${ }^{17}$

\footnotetext{
${ }^{17}$ One might think to diagonalize: We obtain $\omega^{\omega}$ by considering finite iterations of
} 
In the passage quoted above in, Gödel himself seems to suggest that the operation $\alpha \mapsto \alpha^{2}$ preserves graspability, presumably because a path $p$ through $\alpha^{2}$ arises from a path $q$ in $\alpha$ by replacing each node of $q$ by a path through $\alpha$. Of course, assuming the graspability of $\omega$, this operation will yield the same bound $\omega^{\omega}$, but I question Gödel's intuition about this: What $\beta \times \alpha$ amounts to is the construction $--{ }_{\beta}$, but where "- - - now refers, not to arbitrary finite iteration, but rather to iteration up to some arbitrary $\nu<\alpha$. I do not see why the graspability of $\alpha$ should imply the graspability of $--{ }_{\alpha}$. When $\nu$ is infinite, the iteration in question requires the choice of an infinite number of paths through $\beta$. (Once again, it seems to me, Gödel failed to respect the unique character of the finite - this time in finite iteration. $)^{18}$

\section{References}

Ackermann, W. [1924]. Begründung des "tertium non datur" mittels der hilbertschen Theorie der Widerspruchfreiheit, Mathematische Annalen 93: 509-518.

Benacerraf, P. and Putnam, H. (eds) [1964]. Philosophy of Mathematics: Selected Readings, first edn, Cambridge University Press. First edition 1983.

Bernays, P. [1930-31]. Die Philosophie der Mathematik und die Hilbertsche Beweistheorie, Blätter für deutsche Philosophie 4: 326-367. Reprinted in [Bernays, 1976]. A translation by P. Mancosu appears in[Mancosu, 1998], pp. 234-265.

Bernays, P. [1976]. Abhandlungen zur Philosophie der Mathematik, Darmstadt: Wissenschaftliche Buchgesellschaft.

Dedekind, R. [1872]. Stetigkeit und irrationale Zahlen, Braunschweig: Vieweg. in [Dedekind, 1932]. Republished in 1969 by Vieweg and translated in [Dedekind, 1963].

concatenations of paths, where the $n$th path is though $\omega^{n}$. But this requires, not just that each $\omega^{n}$ is graspable, but that we can grasp at one glance the various structural possibilities which exist for paths though any of the infinitely many $\omega^{n}$.

${ }^{18}$ Those who have believed that their 'mathematical IQ' is $\epsilon_{0}$ or, indeed, any ordinal $>\omega^{\omega}$ undoubtedly have let their mental "..." 's represent finite iterations, not of concatenations of paths through some graspable ordinal, but of higher type operations. 
Dedekind, R. [1888]. Was sind und was sollen die Zahlen?', Braunschweig: Vieweg. In Dedekind (1932). Republished in 1969 by Vieweg and translated in [Dedekind, 1963].

Dedekind, R. [1932]. Gesammelte Werke, vol. 3, Braunschweig: Vieweg. Edited by R. Fricke, E. Noether, and O. Ore.

Dedekind, R. [1963]. Essays on the Theory of Numbers, New York: Dover. English translation by W.W. Berman of [Dedekind, 1872] and [Dedekind, 1888].

Ewald, W. (ed.) [1996]. From Kant to Hilbert: A Source Book in the Foundations of Mathematics, Oxford: Oxford University Press. Two volumes.

Friedman, M. [1992]. Kant and the Exact Sciences, Cambridge: Harvard University Press.

Friedman, M. [2008]. Synthetic history reconsidered, in M. Domski and M. Dickson (eds), Essays on a New Method: Reinvigorating the Marriage of History and PHilosophy of Science, Chicago: Open Court Press, pp. 3-190.

Gentzen, G. [1943]. Beweisbarkeit und unbeweisbarkeit von Anfangsfällen der transfiniten induktion in der reinen Zahlentheorie, Mathematische Annalen 119: 140-161.

Gödel, K. [*193?]. Undecidable diophantine propositions, in Collected Works, Vol. III [Gödel, 1995], pp. 164-175.

Gödel, K. [1931]. Über formal unentscheidbare Sätze der Principia Mathematica und verwandter Systeme I, Monatshefte für Mathematik und Physik 38: 173-198.

Gödel, K. [*1933o]. The present situation in the foundations of mathematics, in Collected Works, Vol. III [Gödel, 1995], pp. 45-53.

Gödel, K. [1934]. On undecidable propositions of formal mathematical systems, pp. 346-372. Mimeographed lecture notes by S. Kleene and J. B. Rosser reprinted in [Gödel, 1986].

Gödel, K. [1938a]. Lecture at Zilsel's, in Collected Works, Vol. III [Gödel, 1995], pp. 87-113. 
Gödel, K. [*1941]. In what sense is intuitionistic logic constructive?, in Collected Works, Vol. III [Gödel, 1995], pp. 189-201.

Gödel, K. [1947]. What is Cantor's continuum problem?, American Mathematical Monthly 54: 515-525. Reprinted in [Gödel, 1990]. [Gödel, 1964] is a revised and expanded version.

Gödel, K. [1958]. Über eine bisher noch nicht benützte Erweiterung des finiten Standpunktes, Dialectica 12: 280-287. Reprinted with an Englsh translation in [Gödel, 1990, 240-252]. [Gödel, 1972] is a revised version.

Gödel, K. [*1961/?]. The modern development of the foundations of mathematics in the light of philosophy, in Collected Works, Vol. III [Gödel, 1995], pp. 374-386.

Gödel, K. [1964]. What is Cantor's continuum problem?, in Benacerraf and Putnam [1964], pp. 258-273. Revised and expanded version of [Gödel, 1947]. Reprinted in [Gödel, 1990]. In the second edition of [Benacerraf and Putnam, 1964], the pages are 470-485.

Gödel, K. [1972]. On an extension of finitary mathematics which has not yet been used, in Collected Works, Vol. II [Gödel, 1990], pp. 271-280. Revised version of [Gödel, 1958].

Gödel, K. [1986]. Collected Works, Vol. I, Oxford: Oxford University Press.

Gödel, K. [1990]. Collected Works, Vol. II, Oxford: Oxford University Press.

Gödel, K. [1995]. Collected Works, Vol. III, Oxford: Oxford University Press.

Gödel, K. [2003a]. Collected Works, Vol. IV, Oxford: Oxford University Press.

Gödel, K. [2003b]. Collected Works, Vol. V, Oxford: Oxford University Press.

Hilbert, D. [1926]. Über das Unendliche, Mathematische Annalen 95: 16190. Translated by Stefan Bauer-Mengelberg in [van Heijenoort, 1967, 367-92].

Hilbert, D. [1931]. Die Grundlegung der elementaren Zahlentheorie, Mathematische Annalen 104: 485-94. English translation by W. Ewald in [Ewald, 1996, pp. 1148-57]. 
Hilbert, D. and Bernays, P. [1934]. Grundlagen der Mathematik I, Berlin: Springer-Verlag. The second edition was published in 1968.

Kreisel, G. [1958]. Hilbert's programme, Dialectica 12: 346-372. Reprinted with a Postscript and some notes added in [Benacerraf and Putnam, 1964, pp. 207-238].

Kreisel, G. [1960]. Ordinal logics and the characterization of informal notions of proof, Proceedings of the International Congress of Mathematicians, Edinburgh pp. 289-299.

Kreisel, G. [1965]. Mathematical logic, in T. Saaty (ed.), Lectures on Modern Mathematics, New York: Wiley, pp. 95-195.

Mancosu, P. (ed.) [1998]. From Brouwer to Hilbert: The Debate on the Foundations of Mathematics in the 1920's, Oxford: Oxford University Press.

Parsons, C. [1995]. Platonism and mathematical intuition in Kurt Gödel's thought, Bulletin of Symbolic Logic 1: 44-74.

Parsons, C. [1998]. Finitism and intuitive knowledge, in M. Schirn (ed.), The Philosophy of Mathematics Today, Oxford: Clareendon Press, pp. 249270.

Poincaré, H. [1900]. Du rôle de l'intuition et de la logique en mathématiques, Compte redu du Deuxième congrès international des mathématiciens tenu à Paris du 6 au 12 août 1900 pp. 115-130. Translation by George Bruce Halsted in [Ewald, 1996], Vol. 2, pp 1012-1020.

Sieg, W. [2006]. Gödel on computability, Philosophia Mathematica 14(2): 189-207.

Stein, H. [1988]. Logos, logic and logistiké, pp. 238-59.

Tait, W. [1961]. Nested recursion, Mathematische Annalen 143: 236-250.

Tait, W. [1981]. Finitism, Journal of Philosophy 78: 524-556.

Tait, W. [1986]. Truth and proof: the Platonism of mathematics, Synthese 69: 341-370. 
Tait, W. [2002]. Remarks on finitism, Reflections on the Foundations of Mathematics: Essays in Honor of Solomon Feferman pp. 410-19. Reprinted in [Tait, 2005b], pp. 43-53.

Tait, W. [2005a]. Gödel's interpretation of intuitionism, Philosophia Mathematica .

Tait, W. [2005b]. The Provenance of Pure Reason: Essays in the Philosophy of Mathematics and Its History, Oxford: Oxford University Press.

Tait, W. [2006]. Gödel's correspondence on proof theory and constructive mathematics, Philosophia Mathematica 14: 76-111.

van Heijenoort, J. (ed.) [1967]. From Frege to Gödel: A Source Book in Mathematical Logic, Cambridge: Harvard University Press.

Weyl, H. [1918]. Das Kontinuum: Kritische Untersuchungen über die Grundlagen der Analysis, Leipzig: Veit.

Weyl, H. [1921]. Über die neue Grundlagenkrise der Mathematik, Mathematische Zeitschrift 10: 39-79. Translated by P. Mancosu in [Mancosu, 1998].

Weyl, H. [1949]. Philosophy of Mathematics and Natural Science, Princeton: Princeton University Press.

Zach, R. [1998]. Numbers and functions in Hilbert's finitism, Taiwanese Journal for Philosophy and History of Science 10: 33-60.

Zach, R. [2003]. The practice of finitism: epsilon calculus and consistency proofs in hilbert's program, Synthese 137: 211-259. 